\title{
A Novel Clustering Scheme in Vehicular Ad hoc Network
}

\author{
Amarpreet Singh \\ Associate Processor \\ ACET, Amritsar \\ Amritsar, India
}

\author{
Manverpreet Kaur \\ Research scholar, M.tech \\ ACET, Amritsar \\ Amritsar, India
}

\begin{abstract}
The demand for vehicular ad hoc networks is increasing day by day, since it offers various applications and marvelous benefits to its users. For example, driving safety, entertainment, emergency applications, and data sharing Vehicular networks are identified by their highly dynamic topology. Due to frequent dynamic nature the nodes in VANETs faces connectivity problems. To make this dynamic topology stable during communication, dynamic clustering is found to be one of the best solutions. To overcome such connectivity issues, a novel clustering scheme is presented to keep connectivity maintenance in vehicular ad hoc network. The proposed algorithm aims to improve the cluster lifetime so that vehicles in a cluster can interact with each other for a long time.
\end{abstract}

\section{General Terms}

Clustering Algorithm, Connectivity

\section{Keywords}

VANETs, Clustering, Connectivity, Cluster lifetime, Cluster Head

\section{INTRODUCTION}

Vehicular Ad hoc Network (VANETs) is the assuring approach to give safety and other applications to the people. Vehicular ad-hoc networks are liable for the communication between moving vehicles in a network. Generally, the VANETS consider two types of communication i.e. V2V (Vehicular to Vehicular) in which vehicles can communicate directly with any another vehicle Or V2I (Vehicle-toInfrastructure) in which vehicles can communicate to an infrastructure such as a Road Side Unit (RSU). VANETs provide many services to its users including safety and comport applications Post-Crash Notification, Electronic Toll Collection, and Parking Availability, Alert notifications. For example, vehicles can inform to other vehicles into the network that there is traffic accident or congestion so that the vehicles can change their route immediately. Also, it offers vehicles to connect by Internet to get real time news and weather reports. VANET also provides the huge opportunities to its online users for entertainments like gaming, chatting, multimedia streaming and file sharing via the Internet. The Vehicular Network System is made up of a large number of Vehicles, approximately number of Vehicles above 750 million in the world today. [1] The nodes move in the dynamic network covering the range of 100 to 300 meters to connect to each other and form wireless network. [2]

Although, VANET offers many services to its users but it faces many issues like vehicles have limited transmission range, less storage power. [3] Instead of, these common problems in wireless networks; One main issue is vehicles have high dynamic topology which introduces connectivity problems between the vehicles. To perform communication, all VANETs services must need of connectivity of vehicles in to the network. To overcome this problem; one of the emerging technique is clustering. Clustering is efficient control approach that makes the global network less dynamic. It is best suitable for mobile networks [4] sensor networks [5], and for vehicular networks [6], [7].

In this paper, a new clustering mechanism is introduced to prolonging the cluster lifetime. The idea behind of is cluster the vehicles in a group based on the longest list of neighbors of vehicles. The selection of cluster head depends upon the average speed of vehicles and distance with respect to their respective destination to overcome the fast connection and dis connection event.

Section 2 describes the clustering approach Section 3 Describes the related work done by various investigators Section 3 describes the proposed mechanism Section 4 we conclude the paper.

\section{CLUSTERING}

Clustering is a scheme for grouping vehicles in geographical area together and making the network more efficient and scalable.[8]The method of grouping nodes i.e. mobile devices, sensors, vehicles etc. together is based upon some techniques and it also splits the network into interconnected substructures called clusters. Clusters are a kind of virtual groups that have been developed by applying some clustering algorithm. Each cluster contains at least one cluster Head and two or more cluster members. Inside the cluster one vehicle performs the coordinate task to handle the cluster activities is the Cluster Head. The other node which is also very important is the GW (Gateway node). The gateway node is that node which can hear the communication between the two clusters. The rest of the nodes in a cluster are the ordinary members that directly communicate with cluster head.

\subsection{Types of Clustering}

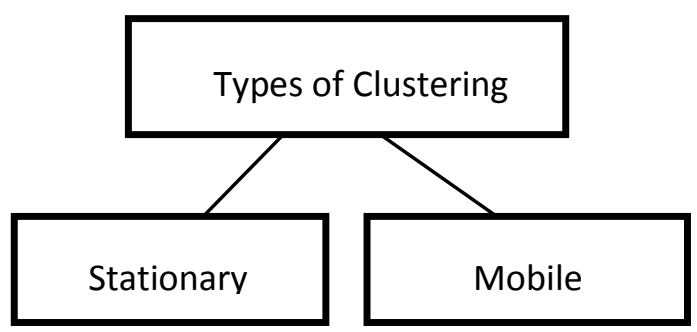

Fig 1: Types of Clustering

\subsubsection{Stationary Cluster}

Stationary Cluster is normally the pre-defined clusters of the geographical region. Sometimes, RSUs (Road Side Units) having fixed infrastructure could also take participate in these types of clusters. In these situations, the cluster range can be equivalent to the transmission range of a RSU. Design of 
routing protocol can be easy to use by using RSU because we do not have need to consider multi-hop situation. But, the fixed RSUs could lead a problem of frequent re-clustering [9]. Frequent re- clustering is not needed because it can cause further cluster formation process of whole network. The physical clustering is only suitable for the applications where vehicles move with moderate speed. But if the vehicles move with high speed, nodes leaving and joining the cluster soon that degrades the stability of the network. [10]

\subsubsection{Mobile cluster}

Mobile clusters are more suitable to handle the mobile features of VANETs since clusters are moving along with vehicles. The mobile cluster can help us to build more stable and effective clusters than the stationary cluster. Because in the mobile clustering $\mathrm{CH}$ (Cluster Head) moves with the vehicles that results less re - clustering process will occur. Though, it spends additional time and bandwidth, which not be avoidable, to calculate suitability of cluster-head.

\section{RELATED WORK}

L. Ahmed et al. [10] Uses a clustering mechanism based for connectivity maintenance in VANET with the thought of virtual mobile clustering to provide information to each other vehicles interchanging their Hello messages i.e. their id, status, longest list of neighbors and their velocity. The idea of cluster head election mechanism is depends upon the average speed of the vehicles lie in the same direction.

M S.Thamarai et al. [11] Uses a cluster based vehicle model for vehicle reliable communication, having vehicle density, speed and position of the vehicles to overcome the delay overhead. The main concept of cluster head chosen is depending on the speed and position of the vehicles. It also introduces the cluster head switching scheme i.e. if any vehicle which enter into the cluster, speed is greater than the cluster head than that vehicle is chosen as the cluster head.

R Y Zaydoun et al. [12] have taken the idea of multi-metric Cluster Head $(\mathrm{CH})$ election technique. Vehicles used this technique to calculate their suitability value. Hence, the vehicle which has connected with a longer period of time with other vehicles, keeping closer distances to their neighbors and closer speed to the average speed of their neighbours has high suitability value to being a $\mathrm{CH}$ (Cluster Head).

M. S. Kakkasageri et al. [13] introduced a dynamic and stable clusters in VANETs by using a multiagent system with vehicle direction, speed, connectivity degree to other vehicles and mobility pattern. The vehicle which has ability to connect with more vehicles with less average speed is more qualified for being a cluster head.

M.Hari Prasad et al. [14] a novel algorithm to construct Stable clusters to achieve cluster based routing. The algorithm groups the nodes based on the position and direction information. The cluster head is calculated by its suitability value u, i.e., calculated based on mobility information of its neighbourhood. The node having higher probability to elect as a cluster Head, which have higher no. of stable neighbours, having closer distances to their neighbours and also having closer speed to the average speed that means they have higher suitability value.

P. Sheth et al. [15] Multi- hop stable clustering in VANETs on highways. The author develops the stable clustering mechanism for the highway scenario. This algorithm performs clustering with direction of vehicle and its average speed. It used the pre-specified speed range and clustered the vehicles according to the speed range. So it has decreased the no. of changes in the cluster heads.

G D.Rajini et al. [16] Uses the location based multipath flooding algorithm to transmit the updated real time information and keep long link duration to improve the data delivery ratio which is suitable for vehicles with variable mobility. It reduces the re clustering process and makes more number of stable clusters than other models.

M C Aswathy et al. [17] studied the dynamic change in topology effects the effective time of routing. Hence routing in VANETs is complex. During the route discovery process AODV broadcasts a route request message (RREQ). It generates many unused routes between a source and a destination node. To improve the performance, the VANET is made into small clusters with long Cluster Head duration. The AODV protocol is improved by exchanging broadcasting by RREQ packets with the forwarding of RREQ packets to Cluster Heads and there by managing routing by Cluster Heads and Gateway Nodes. This paper focus on improving the performance of AODV by enhancing the existing protocol by generating stable clusters and performing routing by Cluster Heads and Gateway nodes

$S \boldsymbol{R}$. Pandi et al. [18] designed a new weight based clustering algorithm to improve the performance in this wireless technology. This clustering algorithm takes the maximum hop distance from the cluster head to its farthest cluster member is two hops which will ensure that each non cluster head node is managed by only one cluster head which are its neighbours within two hops. For weight calculation each node compares the weight of its neighbours. The node which has the largest weight proclaims itself as a cluster head.

From the above write up we observed that the transmission of control messages among vehicles during cluster formation and cluster Head selection increases the chances of routing overhead into the network. Secondly, to choose a $\mathrm{CH}$ which has more ability to stay over road for a long period of time is a big challenge. To overcome these problems, a new clustering approach for the vehicular ad hoc network is introduced. 


\section{PROPOSED SOLUTION}

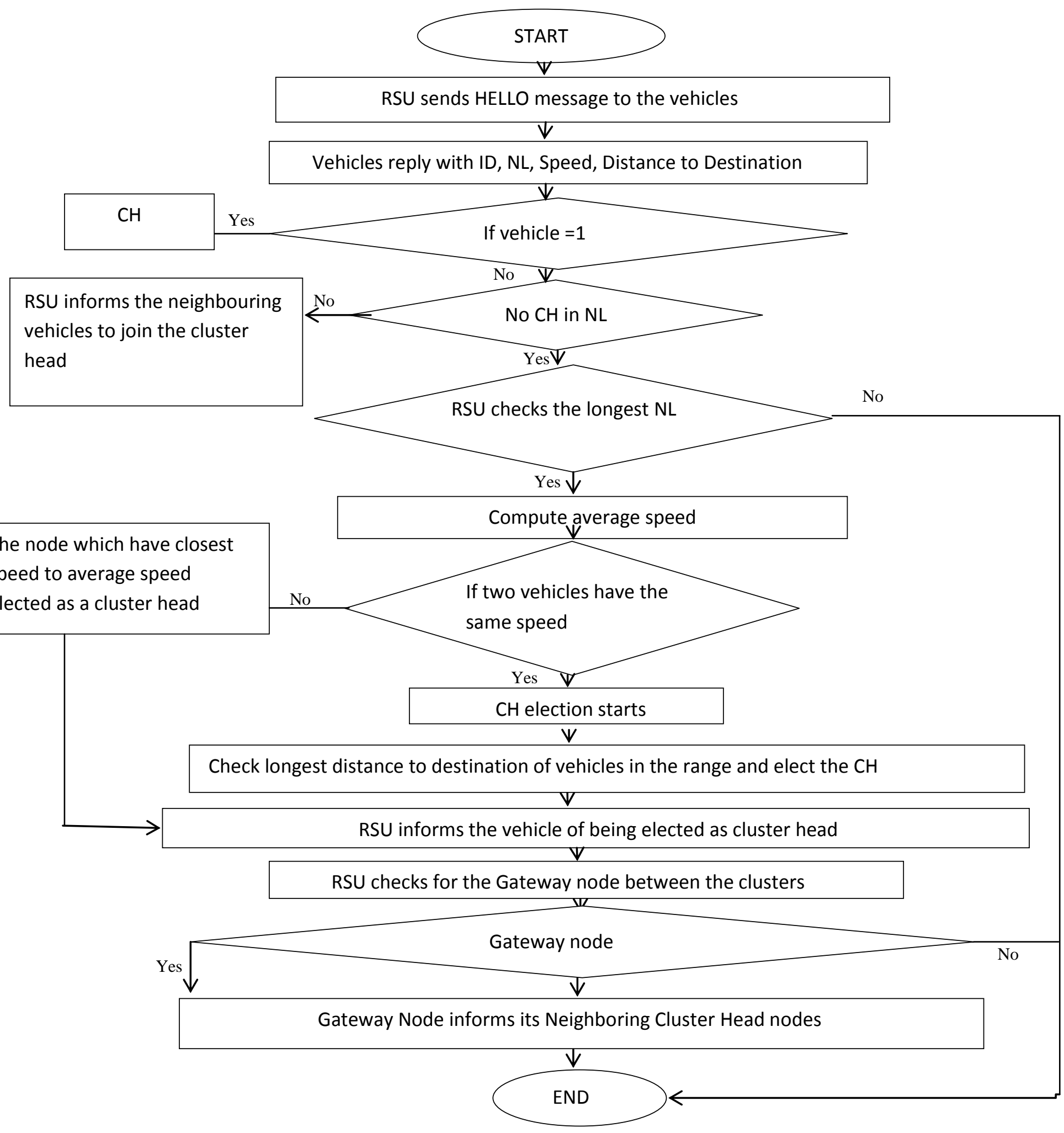

Fig 2: Proposed Flowchart 
This paper introduces the clustering mechanism in which RSU (Road Side Unit) performs the cluster formation and cluster Head selection. RSU sends the Hello messages to all the vehicles. Vehicles reply back to RSU with their ID, NL, Speed, and Distance with respect to their respective destination. Firstly, RSU checks the longest list of neighbors of vehicles for cluster formation. If the vehicle has closest speed to the average speed then it will be elect as a Cluster Head. Otherwise, the vehicle which has longer distance with respect to their respective destination will be electing as a cluster Head. The Gateway node will be also select by the RSU for providing the communication facility between the clusters.

\subsection{Proposed Algorithm}

1. The first step is the deployment of all the vehicles in the network along with the RSU.

2. Cluster formation steps begin with RSU sending the hello messages to each vehicle having same direction. The vehicles upon receiving the hello message check their neighbor list.

3. The vehicles reply back to RSU with their Id, their neighbour list, their speed and the distance to the destination. The speed and destination will be allocated to all the vehicles using the random waypoint mobility model.

4. For the cluster formation, RSU check the longest list of neighbours of each node.

5. The RSU upon receiving the information proceed for the cluster head election process.

A) It computes the average speed of the vehicles and elects the cluster Head, the node which closest speed to average speed elect as a cluster head by the RSU.

$B$ But If two vehicles having the same speed, then, the node with longer distance to destination chosen to be the cluster head.

C) RSU informs the vehicles being elected as a cluster Head.

After the selection of $\mathrm{CH}, \mathrm{CH}$ informs to all vehicles being member of that $\mathrm{CH}$. Moreover, to provide the communication link between two clusters the Gateway nodes are used. Gateway node is a node which is common between two clusters. It helps to provide the communication between two clusters.

\section{CONCLUSION}

In this paper, a clustering scheme for vehicular ad hoc network is introduced. The main goal of proposed technique is to improve the cluster lifetime so that the network stability can be improved. The exchanging of control messages among the vehicles during the cluster formation and cluster Head selection introduce the routing overhead into the network. So, the use of RSU for the cluster formation and cluster Head selection will help to reduce the routing overhead into the network. The selection of cluster Head by average speed of vehicles and distance with respect to their respective destination will help to establish the long living clusters. In the future, this technique can be implemented by using various simulators such as NS2, SUMO, OMNET++ and NS3.

\section{REFERENCES}

[1] M Raya, D Jungels, P Papadimitratos, I Aad, JHubaux, 2006 "Certificate revocation in vehicular networks" laboratory for computer communications and applications school of computer and communication sciences EPFL, Switzerland

[2] Priya Sharma, Amarpreet Singh September 2015 enhanced attacked packet detection algorithm for detecting dos attack in Vanets Proceedings of 38th IRF International Conference, 27th, Pune, India, ISBN: 978 93-85832-03-1

[3] Shyju Raju, D.A Vol.6, No.5, 2015 "Parikh Performance Improvement in VANET by Modifying AODV Routing Protocol", Computer Engineering and Intelligent Systems

[4] A. Amis, R. Prakash, T. Vuong, and H. D.T., 2000 "Max-min d-cluster in wireless ad hoc networks." in Proceedings of the IEEE Conference on Computer Communications (INFOCOM), pp. 32-41.

[5] O. Younis, M. Krunz, and S. Ramasubramanian 2006 , "Node clustering in wireless sensor networks: Recent developments and deployment challenges," IEEE Network magazine, vol. 20, no. 3, pp. 20-25,

[6] L. Maglaras and D. Katsaros, 2012 "Distributed clustering in vehicular networks," in Proceedings of the International Workshop on Vehicular Communications and Networking (VECON),

[7] M. Ni, Z. Zhong, and D. Zhao, 2011 "MPBC: A mobility prediction-based clustering scheme for ad hoc networks," IEEE Transactions on Vehicular Technology, vol. 60, no. 9, pp. 4549-4559,

[8] Manverpreet Kaur, Amarpreet Singh 2015 "Clustering in vehicular ad hoc Network: A Survey" International Journal of Advanced Research in Computer Science, 6 (5), May-June, 138-140

[9] Wang YX, Bao FSh 2007 “An entropy-based weighted clustering algorithm and its optimization for ad hoc networks," Proceedings of the third IEEE international conference on wireless and mobile computing, networking and communications (WiMob2007)

[10] L.Ahmed 2014 Clustering-based Algorithm for Connectivity Maintenance in Vehicular Ad-Hoc Networks IEEE

[11] M .S Thamarai 2013 A Cluster-based Highway Vehicle Communication in VANET International Journal of Computer Applications National Conference on Recent Trends in Computer Applications NCRTCA

[12] ZaydounY. Rawshdeh 2009 toward Strongly Connected Clustering Structure in Vehicular Adhoc Networks IEEE

[13] M. S. Kakkasageri February 2014 Connectivity and Mobility Aware Dynamic Clustering in VANET International Journal of Future Computer and Communication, Vol. 3, No. 1.

[14] M.Hari Prasad Vol.3,Issue5,May2014 Performance Enhancement of VANETs Using Cluster Based Routing International Journal of Innovative Research in Science, Engineering and Technology 
[15] P. Sheth et al. Volume 2, Issue 5, May 2014 Multi- hop stable clustering in VANETs on highways International Journal of Advance Research in Computer Science and Management Studies

[16] G D.Rajini et al. 2010 A Novel Cluster based Routing Algorithm for Hybrid Mobility Model in VANET International Journal of Computer Applications Volume 1 - No. 15
[17] M. C Aswathy Vol.3, No.3, September 2012 cluster based enhancement to AODV for inter- vehicular communication in vanet International Journal of Grid Computing\& Applications (IJGCA)

[18] R. Pandi Selvam et al, Vol.2(2),2011 Stable and Flexible Weight based Clustering Algorithm in Mobile Ad- hoc Networks International Journal of Computer Science and Information Technologies 